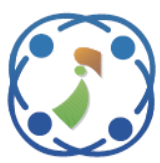

\title{
Multi Agent Based Diabetes Diagnosing and Classification with the Aid of Hybrid Firefly-Neural Network
}

\author{
Kiran Tangod ${ }^{1 *}$ \\ ${ }^{1}$ Gogte Institute of Technology, Belagavi, Karnataka, India \\ ${ }^{2}$ Jain College of Engineering, Belagavi, Karnataka, India \\ * Corresponding author's Email: kirantangod1176@gmail.com
}

Gururaj Kulkarni

\begin{abstract}
A multi agent distributed data mining system for diagnosing diabetes and classification is proposed. Here we are introducing four agents namely user agent, connection agent, updation agent, and security agent. In which each agent performs their own task under the coordination of the connection agent. The user agent collects the user symptoms in order to predict the patient status also the knowledge based of the system. Updation agent is responsible for prescribing drugs for the patient. For secure communication, the proposed technique introduces one security agent between connection agent and updation agent. Here the user symptoms are encrypted by means of advanced encryption standard (AES). Finally, updation agent is classifying the user symptoms and then evaluates the diabetes level with the help of hybrid firefly based neural network algorithm. The performance of the proposed system will acquire with the classification accuracy. The proposed method will be implemented in JAVA platform.
\end{abstract}

Keywords: Multi agent diabetes, Advanced encryption standard, Artificial neural network and firefly optimization.

\section{Introduction}

Agents have demonstrated to have a lot of compensations in the medical domain. Primarily, several investigators utilized agents for monitoring patient's information attained via Body Area Network (BAN) tools [1]. An amount of new studies have reconnoitered the implementation of these technologies to a variety of sports comprising rugby and soccer, in order to more precisely comprehend player function, conditioning and retrieval [2]. The arena of disseminated data mining emphases on emerging effectual algorithms for mining patterns or data from dispersed datasets without the necessity to consolidating them and occasionally without the necessity to disclose them to others [3]. Distributed Data Mining (DDM) aims at extraction useful pattern from distributed assorted data bases [4].

Over numerous years the area of information mining grown with novel approaches and methods to usage our data to abstract a valuable knowledge, mostly utilized for relational databases [5]. Multi agent approaches have been utilized in building numerous efficacious schemes where a set of agents cooperatively work together to accomplish multifaceted tasks [6]. Multi-Agent system (MAS) has inordinate potential aptitudes in DDM due to its distribution, adaptability, openness, and the collaboration, communication, reactivity, selflearning amongst agents. MAS are appropriate for resolving the disseminated, intelligent and collaborative issues [7]. Multi-Agent system (MAS) has inordinate potential aptitudes in DDM due to its distribution, adaptability openness, and the collaboration, reactivity, communication, selflearning between agents. MAS are suitable for solving the distributed, intelligent and collaborative problems [8]. The main encounter in emerging and applying smart grid results is to produce analytics on the distributed information produced in event processing for latency, scale and robustness issues [9]. MAS often deal with complex applications that require distributed problem solving [10].

A term in Data Mining has been familiarized called as "Distributed Data Mining (DDM)", it's a method of accomplishing Data Mining on Distributed 
Data Warehouses over dissimilar remote locations that either comprises the similar information distributed over dissimilar locations or dissimilar information associated with the similar subject [11]. A mobile agent is a running program that can transfer from host to host in a network and fashioned a novel paradigm for information exchange and resource sharing in quickly increasing and recurrently altering computer network [12]. As significance, one among the most extensively utilized method to DDM in business implementations is to implement outdated DM methods to information that have been repossessed from dissimilar sources and stowed in a central information warehouse [13]. Multi-agent systems (MAS) frequently contract with multifaceted implementations that necessitate dispersed problem solving [14]. Multi-agent system (MAS) displays the huge potential if resolves the disseminated data mining. Consequently, this article familiarizes the multi-agent technology to effort to resolve the issue of dispersed data mining [15]. The overall objective of the suggested technique is to diagnosis the diabetes level using multi agent distributed data mining system through secure communication. In order to attain the objective function effective classification and encryption technique is examined in our recommended technique. The main advantage of the recommended technique attains maximum classification rate when compared to the existing technique.

The remainder of the paper is organized as follows: Section 2 provides an overview of related work in the field. In section 3, the present problems are identified. Section 4 narrates the various stages of the work are explored. Section 5 brings out the experimental results and discussions.

\section{Literature Survey}

F. Ruia, et al. [16], have projected a distributed scheme that comprehends the excavation and transport performances with detached vehicles, an excavator and a transporter. To evaluate the productivity of the projected scheme, a simulation atmosphere has been established. Analysis expending the simulator reveals what function factors of the excavator and the transporter have great effects on the productivity.

K. Kouhei, et al. [17], have anticipated a new autonomy-oriented computing-based technique for community mining (AOCCM) from the multi-agent viewpoint in the dispersed atmosphere. In specific, AOCCM uses reactive agents to elite the neighborhood node with the principal structural resemblance as the candidate node, and thus regulate whether it should be additional into local community on the basis of the modularity gain.

J. Xu and C. Tekin [18], we have projected scrupulously solemnize this issue and progress online learning algorithm that permit the agents to accommodatingly learn how to exploit the overall reward in the worldwide feedback situations without swapping any data among themselves we demonstrate that when the agents perceive the worldwide feedback without faults. The dispersed nature of the measured multi-agent scheme results in to functions loss associates with the case where agents can altercation data.

C. Wu, et al. [19], they have projected multi-agent web service outline on the basis of service-oriented architecture for the optimization of medical information quality in the e-healthcare data scheme. On the basis of the design of the multi-agent web service outline, an evolutionary algorithm (EA) for the dynamic optimization of the medical information quality is projected. To validate the projected outline, experimental solutions for a breast cancer case study are delivered. Additionally, to display the unique function of our algorithm, assessment with other works in the literature review will be offered.

R. Cardell-Oliver, et al. [20], we have projected new language for demonstrating circumstances in sensor networks that discourses these encounters. Three algorithms for distinguishing circumstances in pertinent fields are reviewed and altered to sensor networks. In specific, distributed commitment machines are familiarized and established to be the most appropriate algorithm amongst the three for distinguishing conditions in sensor networks.

W. A. Atteya, et al. [21], has presented efficient real-time knowledge base architecture for multiagent based patient diagnostic system for chronic disease management. The projected model progresses the diagnostic knowledge and determines the diseases on the basis of the minimum number of operative tests, thus, giving accurate medical decisions on the basis of cost operative treatments.

Z. Kafal1, et al. [22], has proposed HYDRA: a multi-agent hybrid diagnosis and monitoring architecture that is aimed at helping diabetic patients manage their illness. The model is progressively updated via incoming descriptions about the patients, and permits prediction of possible future values.

From the literature survey, they mainly focused on the diabetes diagnosing through multi agent. The communication between the agents is not secure and the classification rate is also minimum value these are the main drawbacks of the recent research papers. So that the suggested technique is using the effective classification and encryption technique for secure 
diagnosing through agent.

\section{Problem Definition}

MASs provide many potential advantages; they also present many difficult challenges. Here, I present problems inherent in the design and implementation of MASs. The list of challenges includes problems.

$>$ First, how do we formulate, describe, decompose, and allocate problems and synthesize results among a group of intelligent agents?

$>$ Second, how do we enable agents to communicate and interact? What communication languages? How can heterogeneous agents interoperate? What and when can they communicate? How can we find useful agents in an open environment?

$>$ Third, how do we ensure that agents act coherently in making decisions or taking action, accommodating the non-local effects of local decisions and avoiding harmful interactions? How do we ensure the MAS do not become resource bounded? How do we avoid unstable system behavior?

$>$ Fourth, how do we enable individual agents to represent and reason about the actions, plans, and knowledge of other agents to coordinate with them; how do we reason about the state of their coordinated process (for example, initiation and completion)?
Fifth, how do we engineer and constrain practical DAI systems? How do we design technology platforms and development methodologies for MASs?

These are the main drawbacks of various existing work, which motivate us to do the research on multi agent system.

\section{Proposed Methodology}

In this paper we have proposed a multi agent in the distributed data mining system for diagnosing diabetes with the aid of firefly-neural network technique. In our study we are introducing four agents they are user agent, connection agent, updation agent, and security agent. Where each of the agent perform an own task under the coordination of the connection agent; the user agent enable user to input his or her symptoms in order to predict the patient status also the knowledge based of the system. Updation agent is responsible for prescribing drugs for the patient. The user agent will send request to the connection agent will send request to an updation agent. In between that we have introduce one security agent for secure communication between user and updation agent also the rest of the symptoms are used to perform a classification process with the aid of hybrid firefly-neural network technique.

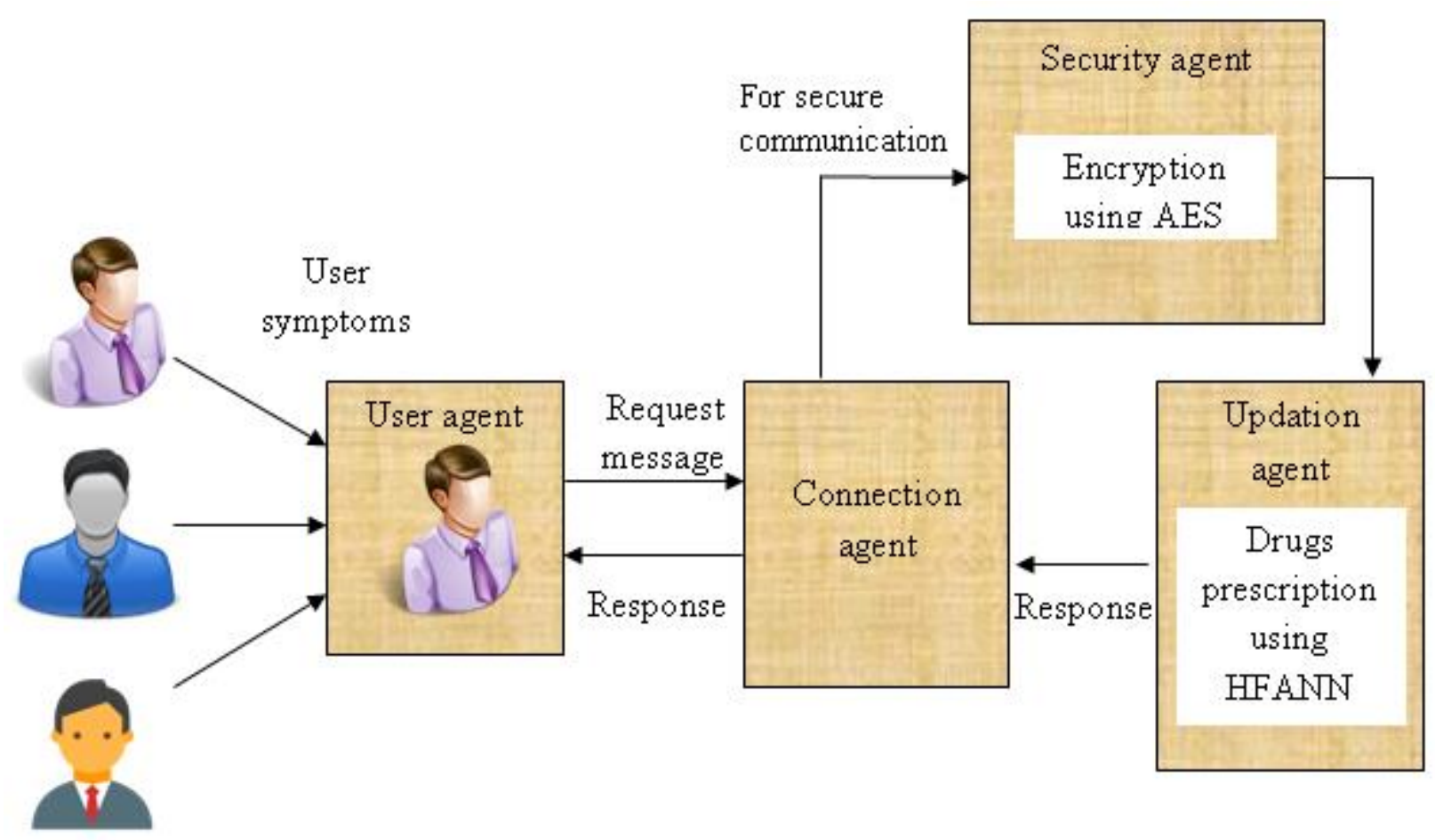

Figure.1 The overall block diagram of the proposed method 
The overall process of the proposed work is shown in fig. 1 and the detail process of the proposed work is illustrated in further section,

The proposed method has four types of agent namely, user agent, connection agent, updation agent and security agent. The details functionality of each agent is described as follows:

User Agent: A user agent asks a connection agent to request its symptoms by sending a request message. It waits to receive the response from the connection agent.

Connection Agent: A connection agent is responsible for connecting the user agent and updation agent. When connection agent receives a request message, it makes the initial connection with updation agent.

Updation Agent: An updation agent is responsible for prescribing drugs for the corresponding user.

Security Agent: A security agent is used for secure communication between user and updation agent.

Initially the user agent collects the user symptoms and then sends the request message to the connection agent. Once the connection agent receives the request message, it makes the initial connection with updation agent. In updation agent, user symptoms are classified and prescribing the drugs based on their diabetes level. For secure communication the proposed methodology introduce the security agent in between the user agent and connection agent, in which cryptography algorithm is used for secure communication. In our proposed method AES (Advanced Encryption Standard) method is used for secure communication. The detailed process of encryption algorithm is illustrated in below section,

\subsection{AES Algorithm}

AES is a block cipher with a block length of 128 bits. It allows three different key lengths: 128, 192 , or 256 bits. We propose AES with 128 bit key length. The encryption process consists of 10 rounds of processing for 128-bit keys. Except for the last round in each case, all other rounds are identical. The $4 \times 4$ matrix of bytes made from 128-bit input block is referred to as the state array. The different transformation operates on the intermediate results, known as state; the state is basically in the form of rectangular array of bytes. Before any round-based processing for encryption can begin, input state is XOR with the first four words of the schedule. The overall process of AES algorithm is shown in fig.2,

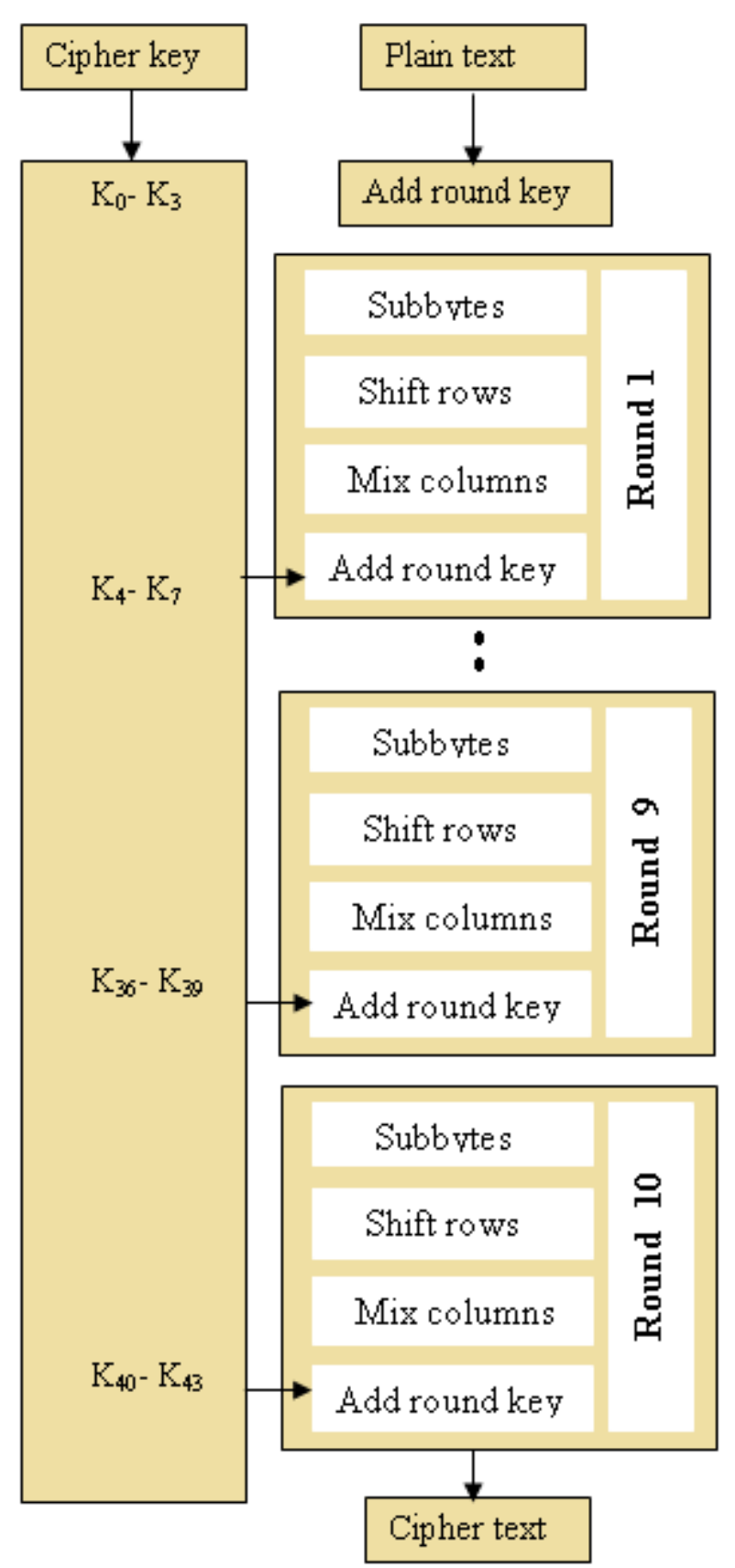

Figure.2 The encryption process of AES algorithm

A state of the proposed work is represent as given in table 1 and A key value of the proposed work is represent as given in table 2 ,

Table 1. A state of proposed work

\begin{tabular}{|l|l|l|l|}
\hline$S O, 0$ & $S 0,1$ & $S 0,2$ & $S O, 3$ \\
\hline$S 1,0$ & $S 1,1$ & $S 1,2$ & $S 1,3$ \\
\hline$S 2,0$ & $S 2,1$ & $S 2,2$ & $S 2,3$ \\
\hline$S 3,0$ & $S 3,1$ & $S 3,2$ & $S 3,3$ \\
\hline
\end{tabular}

DOI: $10.22266 /$ ijies2017.0430.08 


Table 2. A key value of proposed work
\begin{tabular}{|l|l|l|l|}
\hline$K 0,0$ & $K 0,1$ & $K 0,2$ & $K 0,3$ \\
\hline$K 1,0$ & $K 1,1$ & $K 1,2$ & $K 1,3$ \\
\hline$K 2,0$ & $K 2,1$ & $K 2,2$ & $K 2,3$ \\
\hline$K 3,0$ & $K 3,1$ & $K 3,2$ & $K 3,3$ \\
\hline
\end{tabular}

\section{Encryption}

For encryption, each round consists of the following four steps:

- Sub Bytes

- Shift Rows

- Mix Columns

- Add Round Key

\section{Sub-bytes Operation}

The Sub-bytes operation is a non-linear byte substitution, operates on each byte of the state independently. The substitution table (S-Box) is invertible and it is constructed using two transformations.

(i)Take the multiplicative inverse in Rijindael's finite field

(ii)Apply the affine transformation, they have been documented in the Rijindeal documentation.

We utilize the pre-calculation, when the S-box is independent of any input. Then, we substitute the each byte of the state in the s-box whose index corresponds to the value in the state $S(i, j)=\operatorname{SBox}[s(i, j)]$.

\section{Shift Row Operation}

In shift row operation, every row of the state is cyclically shifted to the left which depends on the row index.

$>1$ st row to 0 positions to the left

$>2$ nd row to 1 position to the left

$>$ 3rd row to 2 positions to the left

$>$ 4th row to 3 positions to the left

\section{Mix-Column Operation}

The Mix Columns transformation operates on the State column-by-column, treating each column as a four-term polynomial. The purpose of this step is to provide diffusion of the bits over multiple rounds. This is achieved by performing multiplication one column at a time. Each value in the column is multiplied against every row value of a standard matrix.

\section{Add Round Key}

In Add Round Key, we apply a round key to the state by bitwise XOR. The round key can be derived from the cipher key using key schedule.

Simply it can be written as $C_{i j}=S_{i j} X O R K_{i j}$

From that process, we encrypt the user symptoms and send the encrypted data to the updation agent. In updation agent the decryption process is carried out and then prescribes the drugs based on the user symptoms.

\section{Decryption}

In decryption mode, the operations are in reverse order compared to their order in encryption mode. Thus it starts with an initial round, followed by 9 iterations of an inverse normal round and ends with an Add Round Key. An inverse normal round consists of the following operations in this order: Add Round Key, Inv Mix Columns, Inv Shift Rows, and Inv Sub Bytes. After the decryption process, the updation agent need to find the diabetes level of user if it is normal or abnormal based on that only the updation agent prescribe the drugs for the corresponding user. So that our proposed technique uses the hybrid firefly neural network algorithm is used to classify the diabetes. The detail process of hybrid firefly neural network algorithm is illustrated in below,

\subsection{Hybrid Firefly Neural Network}

The innovative approach employs the artificial neural network (ANN) for medical diagnosis and classification, with the ultimate objective of categorizing the data into two categories such as the normal or abnormal. Further the brand of ANN employed in the suggested technique is the back propagation (BP) approach. Thus, the ANN based diagnosis and classification system emerges as an effective solution for amorphous network data.

\section{Artificial Neural Network using Back Propagation Algorithm}

Artificial neural network based back propagation algorithm is trained using input data. The neural network consists of $\mathrm{n}$ number of input units, $\mathrm{h}$ hidden units and one output unit. Here back propagation algorithm is used as the training algorithm. Here the input data is considered as the user symptoms. The proposed method uses the hybrid firefly algorithm with artificial neural network, in which firefly algorithm is used for weight updation. The structure of artificial neural network is given below. 


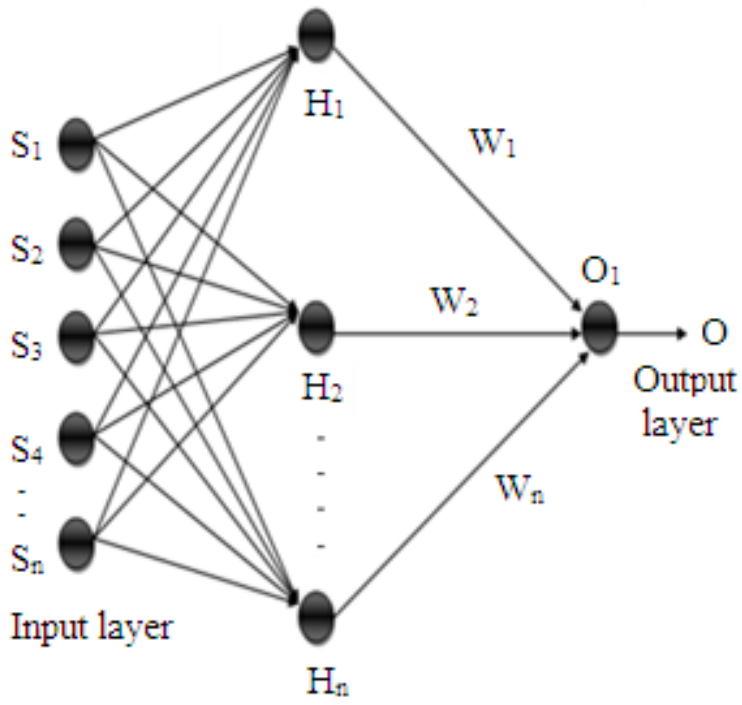

Hidden layer

Figure. 3 The structure of artificial neural network

\section{The Bias Function of the Neural Network}

The Bias Function $\left(B F_{t}\right)$ represents the product of weights and input data of the neural network as illustrated in the following Equation,

$$
B F(t)=\alpha+\sum_{i=1}^{n}\left(w_{11} S_{1}+w_{12} S_{2}+\ldots \ldots . .+w_{t n} S_{n}\right)
$$

Here, $\left(w_{11}, w_{12}, \ldots w_{t n}\right)$ characterize the weights of neuron and $\left(S_{1}, S_{2} \ldots S_{n}\right)$ depict the input data.

\section{Weight Updation using Firefly Algorithm}

In the innovate technique, we deploy the firefly algorithm to update the weights in neural network. Here, initially each weight is arbitrarily arrived at within a definite search space. Further each solution is characterized as weights $w_{i n}$, where $n=(1,2 \ldots m)$. At first, the fitness value of each solution is estimated. The solutions with minimum error values are shortlisted as the current best weights.

$$
\text { fitness }=\min \sum_{n=1}^{m} M S E
$$

Where, MSE represents the mean square error. We estimate the fitness values of each solution. The fitness value of each $i$ th solution is analyzed and contrasted with the $j$ th neighboring solution. If the fitness value of neighboring solution is found higher, we estimate the distance between every solution by means of the Euclidean distance measure. Now, the distance is employed to estimate the attractiveness (A).

$$
A=A_{0} e^{-\beta d_{i j}^{2}}
$$

Where,

$A_{0}$ - refers to the preset attractiveness

$\beta$ - represents the light absorption coefficient

$d_{i j}$ - denotes the distance between $i$ th solution or weight and $j$ th neighboring solution or weight

The new attractiveness value is employed to update the original rules with the help of Equation (4).

$$
w_{i n}^{n e w}=w_{i n}+A\left(w_{j n}-w_{i n}\right)+\alpha(\delta-1 / 2)
$$

Here, $\alpha$ and $\delta$ denote the evenly distributed values between $[0,1] ; w_{\text {in }}$ represents the current weight; $w_{j n}$ represent the $j$ th value except the current weight; therefore, the updated attractiveness values encourage the weight to inch towards the current best fitness value. If the fitness value of the new weights or solution is superior to the original, it gets substituted by the new weights. Or else, the original weight continues to be in the population for the subsequent iteration. The total process gets repeated till the satisfaction of the stopping benchmark. The entire process is elegantly exhibited in the flowchart Fig.4.

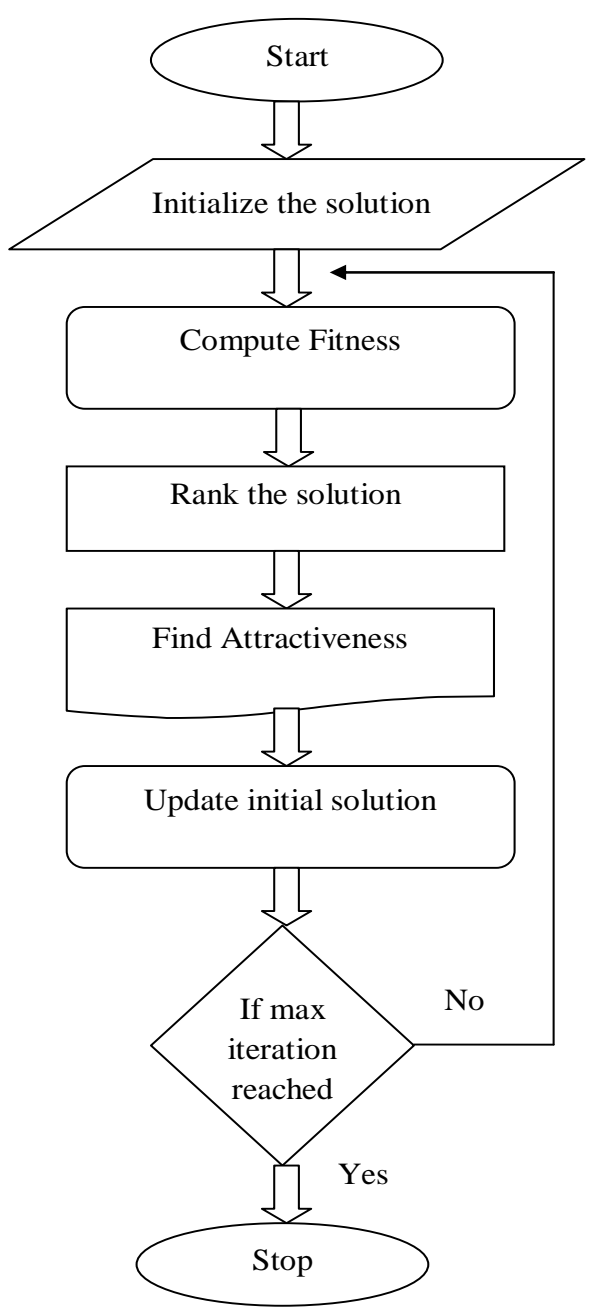

Figure.4 The flowchart for firefly algorithm 
After that we obtain the optimal weights and then the optimal weights are used for further process in neural network.

\section{Activation Functions for the Neural Network}

The Activation function is represented as a nonlinear function as described in the Equation shown below.

$$
H=\frac{1}{1+\exp ^{-B F(t)}}
$$

\section{Calculation of Learning Error obtained is given below,}

The learning error is achieved by means of the following Equation.

$$
O=\frac{1}{2} \sum_{n=0}^{h-1}(\text { desired }- \text { actua })^{2}
$$

Here, $O$ corresponds to the neural network output and $h$ signifies the total number of neurons in the hidden layer.

In the artificial neural network, the error is cutback considerably to the extent of the least value in order that it is well-educated for carrying out the testing phase. Further the threshold value which fulfils the minimum benchmark is allocated. Thereafter the threshold value $\omega$ and the output of the neural network $(O)$ are assessed and contrasted by means of the following equation.

$$
\text { result }=\left\{\begin{array}{l}
\text { normal, } O<\omega, \\
\text { abnormal, } O \geq \omega
\end{array}\right.
$$

If the upshot of the neural network output exceeds the threshold value it indicates that the offered input data is abnormal, or else it represents the normal. Subsequent to the categorization of the input data in to normal or abnormal, based on the classification result the updation agent prescribe the drugs for the corresponding user. The performance of the proposed work is evaluated in section.5. It is illustrated in further section,

\section{Results and Discussion}

This section gives the detailed view of the result that is obtained by our proposed method of multi agent based diabetes diagnosing and classification is performed in the working platform of JAVA. In order to improve the classification accuracy, the proposed technique uses the hybrid firefly neural network. To develop a secure communication through agent, here we are introducing AES encryption algorithm. The experimental result and the performance of the proposed method are given below in detail.

\subsection{Dataset Description}

The proposed method is experimented with the Cleveland dataset. This dataset is taken from the UCI machine learning repository.

\section{Cleveland Data}

This data base contains 76 characteristics, however all distributed tests refer to utilizing a subset of 14 of them. Specially, ML researchers use only the Cleveland database till today. The "goal" field refers to the presence of heart disease in the patient. It is integer valued from 0 (no presence) to 4 . Experiments with the Cleveland database have concentrated on simply attempting to distinguish presence (values 1 , $2,3,4$ ) from absence (value 0 ). The names and social security numbers of the patients were recently removed from the database, replaced with dummy values. Six of the examples have been discarded because they had missing values. Class distributions are $54 \%$ heart disease absent, $46 \%$ heart disease present.

\subsection{Performance Analysis}

The performance analysis of our proposed technique is shown in the below section. Here the table. 3 shows various iteration and the corresponding encryption and decryption time. In our method we take the number of iteration as 10,20,30 and 40. To encrypt the user symptoms our proposed method takes 2561 milliseconds for encryption and 1265 milliseconds for decryption. By varying the number of iteration like 20, 30 and 40 encryption time and decryption time is also varying. Here $2415 \mathrm{~ms}$, $2348 \mathrm{~ms}$ and $2465 \mathrm{~ms}$ to take for encryption and $1145 \mathrm{~ms}, 1063 \mathrm{~ms}$ and $1154 \mathrm{~ms}$ to obtain for decryption by varying the iteration 20,30 and 40 .

Table 3. Encryption and decryption time for various

\begin{tabular}{|c|c|c|}
\hline $\begin{array}{c}\text { No of } \\
\text { iteration }\end{array}$ & $\begin{array}{c}\text { Encryption } \\
\text { time }\end{array}$ & $\begin{array}{c}\text { Decryption } \\
\text { time }\end{array}$ \\
\hline 10 & 2561 & 1265 \\
\hline 20 & 2415 & 1145 \\
\hline 30 & 2348 & 1063 \\
\hline 40 & 2465 & 1154 \\
\hline
\end{tabular}

In our proposed technique Fig.5 and fig.6 shows the overall memory value and execution time of the proposed method. It is plotted in the below section,

The overall memory value of proposed method 
achieves 20597463 bit by varying the number of iteration, the memory value is varying for number of iteration. The overall execution time of the proposed methods achieves 2638414 milliseconds. Fig.6 shows the execution time for the proposed method by varying the number of iteration.

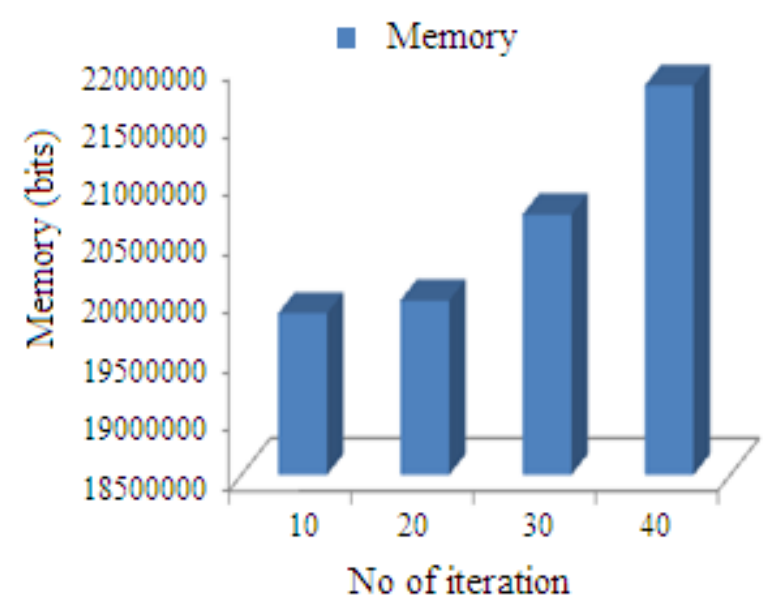

Figure.5 Memory value for the proposed technique

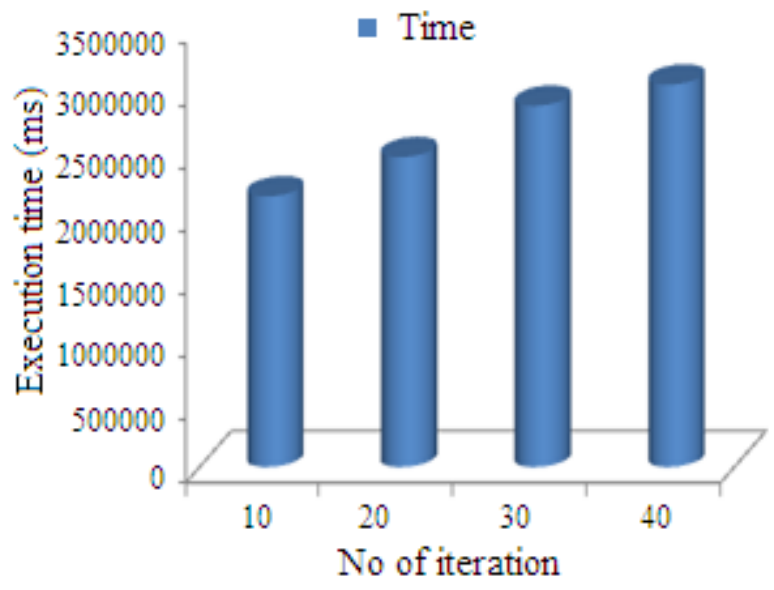

Figure.6 Execution time for the proposed technique

Table 4. Accuracy value of proposed method by varying the iteration

\begin{tabular}{|c|c|}
\hline No of iterations & Accuracy (\%) \\
\hline 10 & 75.12 \\
\hline 20 & 76.34 \\
\hline 30 & 79.14 \\
\hline 40 & 80.47 \\
\hline
\end{tabular}

The overall classification accuracy of the proposed hybrid firefly artificial neural network based back propagation algorithm is tabulated in table.4. Here the proposed ANN achieves $77.7 \%$ of the accuracy value. It is tabulated in beneath,

By varying the number of iteration the proposed classification accuracy also changes. In our proposed hybrid firefly based artificial neural network achieves the accuracy value for the $10^{\text {th }}$ iteration is $75.12 \%$ and the $20^{\text {th }}$ iteration attains $76.34 \%$ and $30^{\text {th }}$ iteration gets the classification accuracy is $79.14 \%$ and also $40^{\text {th }}$ iteration achieves $80.47 \%$ of classification accuracy. From the result we analyze that if the proposed technique reaches the maximum iteration it achieves better classification accuracy.

\subsection{Comparative Analysis}

The comparative analysis of our proposed method is compared with the existing method is tabulated and the result are plotted given below. Table. 4 shows the classification accuracy of the proposed method compared with existing method [23]. In existing method [23] the diabetes classification is detected with the help of fuzzy classifier. The method [23] achieves $75 \%$ of accuracy value. The graphical representation of the proposed comparative analysis for the classification rate is plotted in Fig.7.

In the past studies [23], they use fuzzy classifier for diabetes classification. But in our recommended technique, use hybrid firefly based neural network is used to for diabetes classification. Here the traditional neural network is modified with the help of firefly algorithm. In our recommended technique attains the maximum classification accuracy of $77.7 \%$ which is maximum value when compared to the existing fuzzy classifier [23].

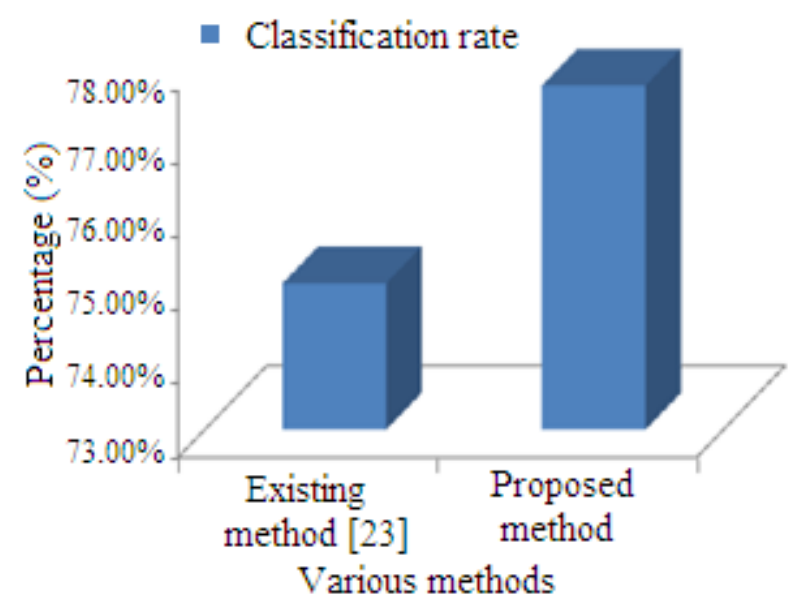

Figure.7 Comparative analysis of classification rate

From the result the suggested technique attains the maximum accuracy because the proposed Hybrid 
Firefly NN reach the minimum error value when compared to the other classifier.

\section{Conclusion}

Multi agent based diabetes diagnosis and classification with aid of hybrid firefly neural network algorithm is proposed in this paper. The proposed technique is implemented in JAVA platform. The performance of the proposed technique is evaluated by classification accuracy, execution time and memory value. The classification accuracy of the proposed technique is compared with existing technique. From the result, the classification accuracy of the implemented technique is high when compared to the existing classifier technique. The proposed multi agent based diabetes diagnosis achieves the maximum classification accuracy of $77.7 \%$. In future the researcher will have sufficient opportunities to perform effective classifier in order to improve the classification accuracy and produce newer heights of excellence in performance.

\section{References}

[1] W. A. Atteya, K. Dahal, "Multi-agent system for early prediction of urinary bladder inflammation disease", In Proceedings of IEEE International conference on Intelligent Systems Designs and Applications, pp. 539-544, 2010.

[2] H. F. Jelineka, A. Kelareva, "Using metaregression data mining to improve predictions ofperformance based on heart rate dynamics for Australian football", ELSEVIER Journal ofApplied Soft Computing, Vol. 14, pp. 81-87, jan 2014.

[3] Y. Kokkinos, K. G. Margaritis, "Confidence ratio affinity propagation in ensemble selection of neural network classifiers for distributed privacypreserving data mining", ELSEVIER Journal of Neuro Computing, Vol. 150, pp. 513-528, Feb 2015.

[4] V. Gorodetsky, O. Karsaeyv, "Multi-agent Technology for Distributed Data Mining and Classification", In Proceedings of ELSEVIER International Conference on Intelligent Agent Technology, pp. 438-441, Oct 2003.

[5] B. Othmane1, R. S. A. Hebri, "Cloud Computing \& Multi-Agent Systems: A New Promising Approach for Distributed Data Mining", In Proceedings of IEEE International Conference on Information Technology Interface (ITI), June 2012.

[6] D. Sharma, F. Shadabi, "Multi-Agents Based Data Mining for Intelligent Decision Support
Systems", In Proceedings of IEEE International Conference on System and Information, pp. 241245, Nov 2014.

[7] D. Zhou, W. Rao, "A Multi-Agent Distributed Data Mining Model based on Algorithm Analysis and Task Prediction", In Proceedings of IEEE International Conference on Information Engineering and Computer Science, pp. 1-4, Dec 2010.

[8] D. Zhou, W. Rao, "A Multi-Agent Distributed Data Mining Model based on Algorithm Analysis and Task Prediction", In Proceedings of IEEE Information Engineering and Computer Science (ICIECS), pp. 1-4, Dec 2010.

[9] D. D. Sharmaa, S. N. Singha, J. Linb, "Multiagent based distributed control of distributed energy storages using load data", ELSEVIER Journal of Energy Storage, pp. 1-12, march 2016.

[10] L. Xiang, "An Agent-based Architecture for Supply Chain Finance Cooperative Contextaware Distributed Data Mining Systems", In Proceedings of IEEE International Conference on Internet web Applications and Service, pp. 261-266, june 2008.

[11] D. Khan, H. Bank, "CAKE - Classifying, Associating \& Knowledge DiscovEry An Approach for Distributed Data Mining (DDM) Using PArallel Data Mining Agents (PADMAs)", In Proceedings of IEEE International Conference on Web Intelligent Agent Technology, Vol. 3, pp. 596-601, Dec 2008.

[12] U. P. Kulkarni, K.K. Tangod, "Exploring the Capabilities of Mobile Agents in Distributed Data Mining", In Proceedings of IEEE International Conference on Engineering and Application, pp. 277-280, Dec 2006.

[13] M. Klusch, S. Lodi, "The Role of Agents in Distributed Data Mining: Issues and Benefits", In Proceedings of IEEE International Conference on Intelligent Agent Technology, pp. 211-217, Oct 2003.

[14] C. Josenildo, Da. Silvaa, C. Giannella, "Distributed data mining and agents", ELSEVIER Journal of Engineering Application of Artificaial Intelligence, Vol. 18, No. 7, pp. 791-807.

[15] Z. Chen"12, S. LiuI, "The Multi-Agent Knowledge Management System Model for Pervasive Computing", In Proceedings of IEEE International Conference on Pervasive Computing and Application, Vol. 1, pp. 70-73, Oct 2008.

[16] F. Ruia, Z. Bu, Z. Wu, "Local Community Mining on Distributed and Dynamic Networks From a Multiagent Perspective", In Proceedings 
of IEEE Transaction on Cybernetics, Vol. 46, No. 4, pp. 986-999, April 2016.

[17] K. Kouhei, "Production analysis of functionally distributed machines for underground mining", ELSEVIER Journal of International Journal of Mining Science and Technology, pp. 477-485, Vol. 26, No. 3, May 2016.

[18] J. Xu, C. Tekin, "Distributed Multi-Agent Online Learning Based on Global Feedback", In Proceedings of IEEE Transaction on Signal Processing, Vol. 63, No. 9, pp. 2225-2238, Feb 2015.

[19] C. S. Wu, Ibrahim Khoury, "Optimizing Medical Data Quality Based on Multiagent Web Service Framework", In Proceedings of IEEE Transaction on Information Technology in Biomedical, Vol. 16, No. 4, pp. 745-757, July 2012.

[20] R. C. Oliver and W. Liu, "Representation and Recognition of Situations in Sensor Networks",
In Proceedings of IEEE Communication Magazine, Vol. 48, No. 3, pp. 112-117, March 2010.

[21] W. A. Atteya, Keshav Dahal, "Multi-agent system for early prediction of urinary bladder inflammation disease", In Proceedings of IEEE International Journal of Intelligent Systems Design and Application, pp. 539-544, 2010.

[22] O. zgür Kafalı, U. Schaechtle, "HYDRA: a Hybrid Diagnosis and monitoring Architecture for diabetes", In Proceedings of IEEE International Conferenc eon e-Health Networking, Applications and Service (Health corn), pp. 531-536, 2014.

[23] A. A. Obiniyi and M. K. Ahmed, "MultiAgent based Diagnostic Model for Diabetes", International journal of scientifica and engineering research, Vol. 6, No. 5, pp. 15891594, May 2015. 